\title{
Direct investigation of the fate of NAPL contaminations in a hydrating cement matrix by means of magnetic resonance techniques
}

by

\author{
Nikolaus Nestle ${ }^{*}, 1,2$, Petrik Galvosas ${ }^{2}$, Christian Zimmermann ${ }^{3}$, Frank Stallmach $^{2}$, Jörg \\ Kärger $^{2}$
}

*,1: Corresponding author: Present address: TU Darmstadt, Institut für Festkörperphysik, Hochschulstraße 6, D-64289 Darmstadt, Germany. phone: ++49 615116 2934, FAX ++ 49 615116 2833, e-mail: nikolaus.nestle@physik.tu-darmstadt.de

${ }^{2}$ Universität Leipzig, Abteilung Grenzflächenphysik, Linnéstraße 5, D-04103 Leipzig, Germany,

${ }^{3}$ IdM an der Universität Ulm, D-89069 Ulm, Germany

Supporting information comprises 1 Table and 4 figures on 4 pages including cover. 


\begin{tabular}{|l|r|r|r|r|r|r|r|r|r|}
\hline & $A_{o}[1 / \mathrm{s}]$ & error & $m$ & error & $K[1 / \mathrm{s}]$ & error & $1 / T_{2 \mathrm{o}}[1 / \mathrm{s}]$ & error & $T_{2 \mathrm{o}}[\mathrm{ms}]$ \\
\hline Toluene & 1841 & 8 & 2.001 & 0.019 & 0.00048 & 0.00003 & 449 & 4 & 2.23 \\
\hline Cyclooctane & 1009 & 5 & 1.951 & 0.027 & 0.00117 & 0.00012 & 427 & 5 & 2.34 \\
\hline n-Hexanol & 1004 & 8 & 1.518 & 0.022 & 0.00354 & 0.00029 & 375 & 5 & 2.66 \\
\hline
\end{tabular}

Table S1. Complete fitting results for the Avrami-type kinetic model (Eqn. 2) to the data shown in figure 2 .

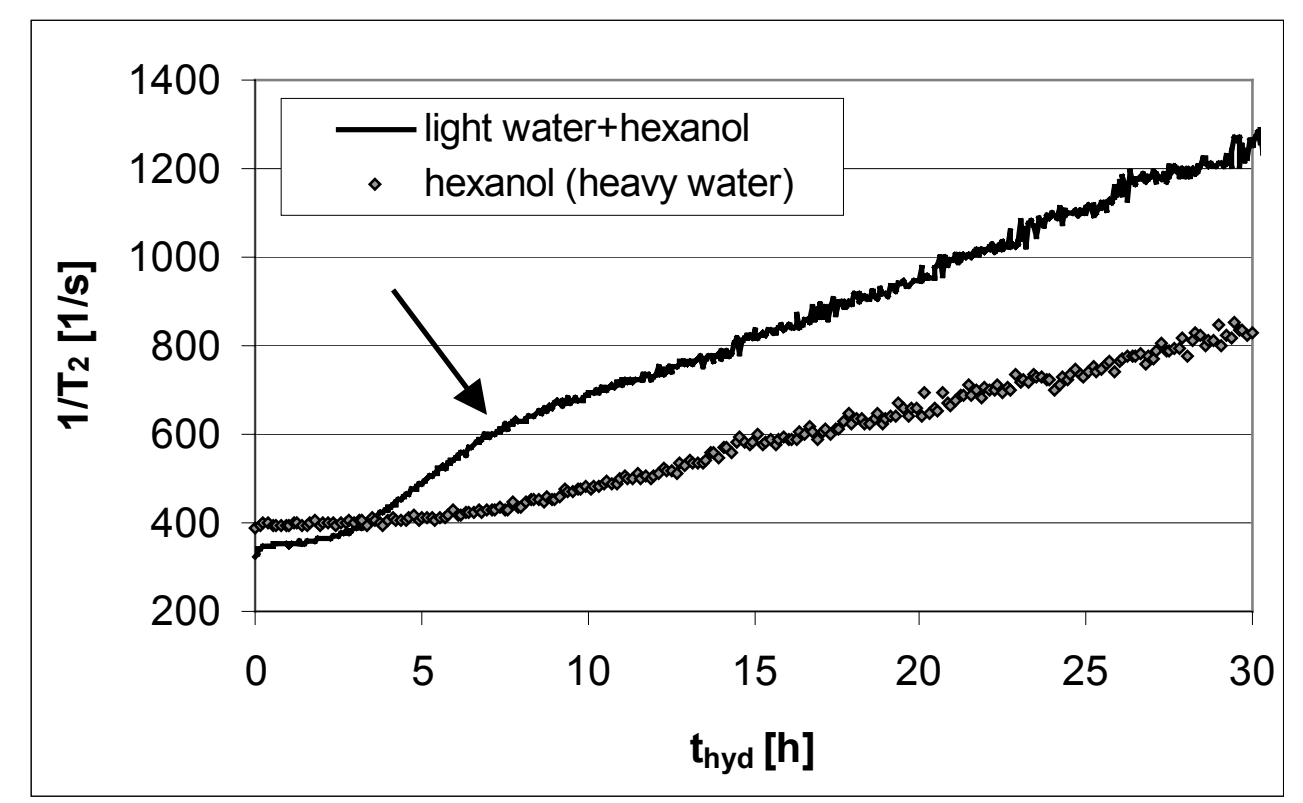

Figure S1. Proton transverse relaxation rates in hydrating ordinary and deuterated white cement at w/c 0.33 with added hexanol. Note the change in the slope of the relaxation rate of the water marked with an arrow. This structure might be related to the marked structure in the diffusion data in fig. 6 in the paper. 


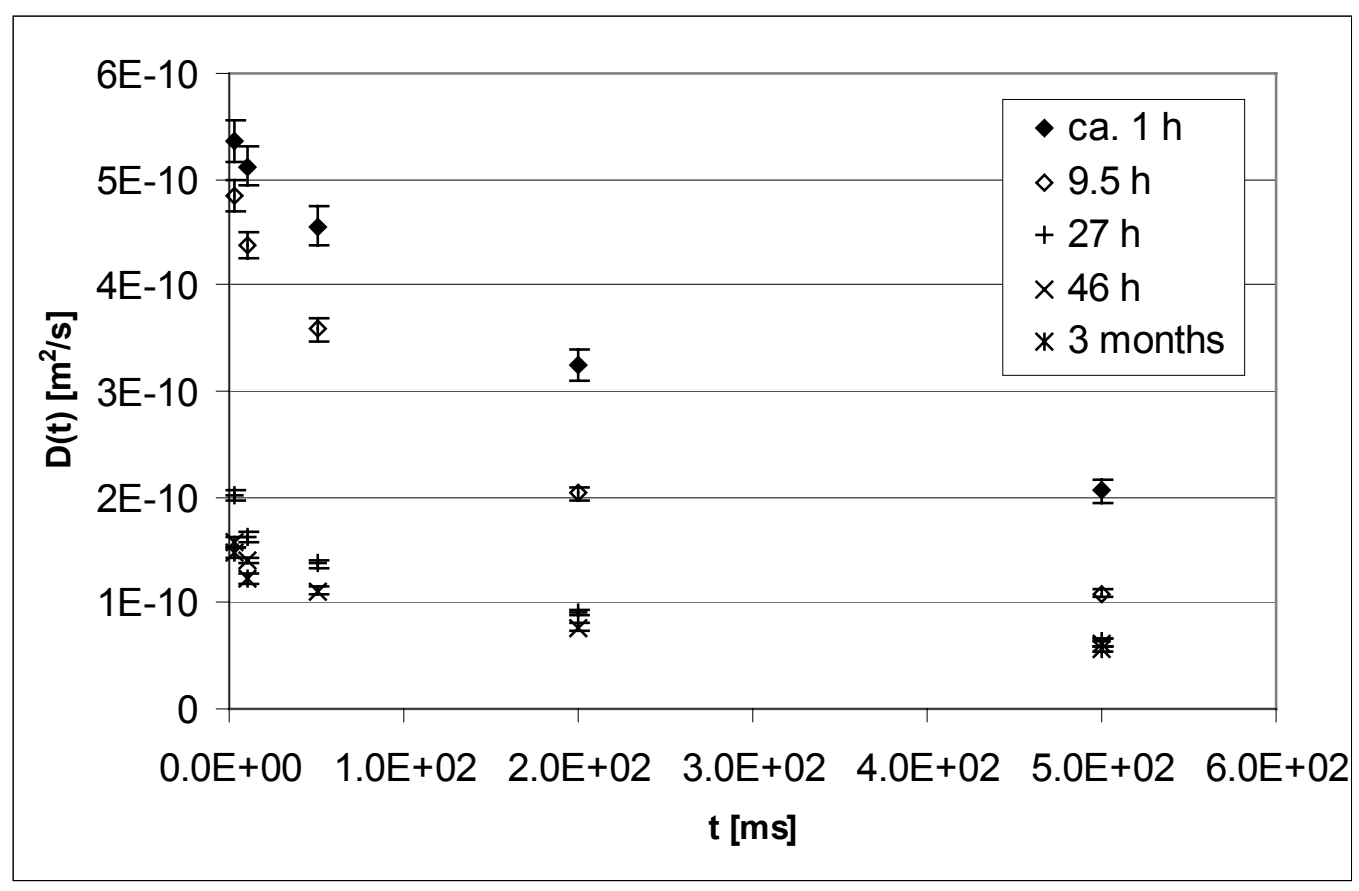

Figure S2. The diffusion data of figure 7 in the paper as a function of the observation time.

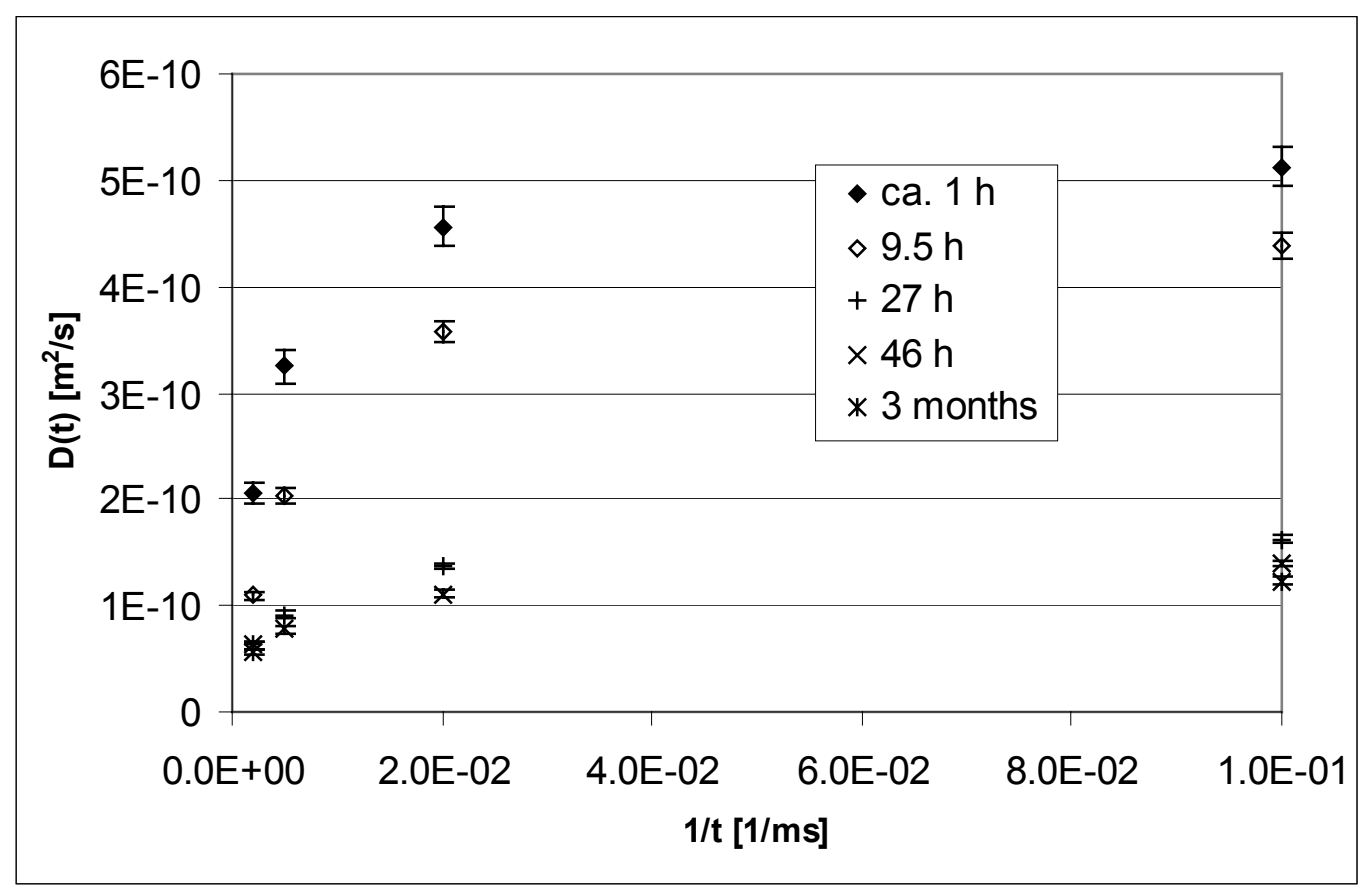

Figure S3. Diffusion data of figure 7 in the paper as a function of the reciprocal observation time. For none of the hydration times, the diffusion coefficients are found to be directly proportional to the reciprocal observation time. In all cases, the approximation to infinite observation times still leads to a finite value diffusion coefficient. 


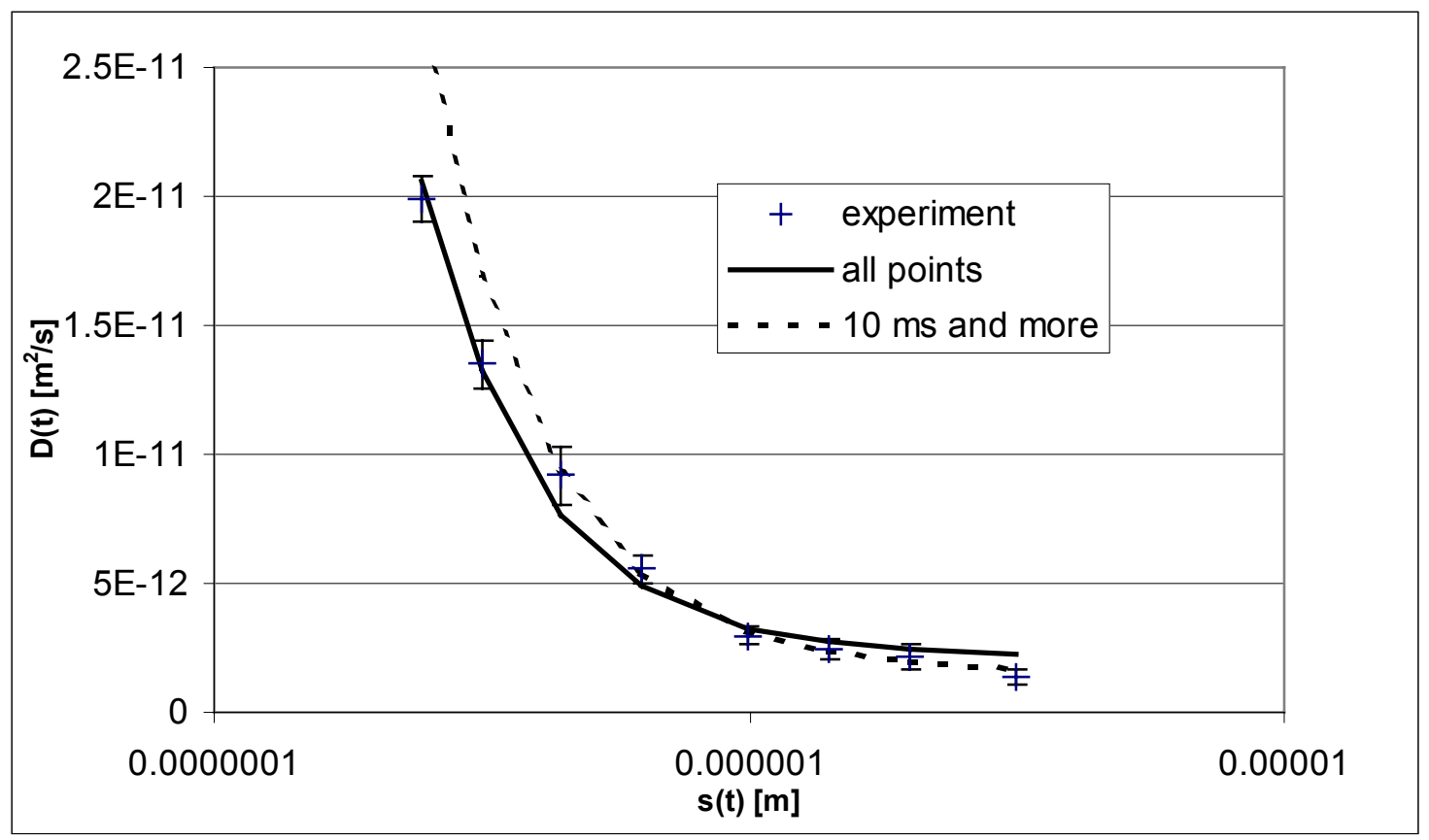

Figure S4. Diffusion data of figure 8 in the paper as a function of the mean diffusive shift computed from the short-time diffusion coefficient and the observation time. The lines in the graph are the same fitting curves as in figure 8 in the paper. 